\title{
Effect of L-tryptophan in promoting the kinetics of carbon dioxide hydrate
}

formation

Himanshu Khandelwal ${ }^{1}$, M Fahed Qureshi ${ }^{1}$, Junjie Zheng ${ }^{1}$, Pradeep Venkataraman ${ }^{2}$, Timothy A Barckholtz ${ }^{3}$, Ashish B Mhadeshwar ${ }^{3}$, Praveen Linga ${ }^{1, *}$

${ }^{I}$ Department of Chemical and Biomolecular Engineering, National University of Singapore, Singapore 117 585, Singapore

${ }^{2}$ ExxonMobil Upstream Research Company, Houston, TX, USA

${ }^{3}$ ExxonMobil Research and Engineering, Annandale, NJ, USA

\section{Supplementary material:}

2 Tables

4 Figures

6 Videos

Corresponding author(s): praveen.linga@nus.edu.sg (P. Linga) 


\section{S.1 Fugacity driving force calculation}

Fugacity at a particular pressure and temperature is calculated by applying the Peng-Robinson equation of state. Equation for calculating fugacity follows as

$$
f=P * \exp \left(Z-1-\ln (Z-B)-\frac{A}{2 \sqrt{2 B}} \ln \left(\frac{Z+2.414 B}{Z-0.414 B}\right)\right) \ldots
$$

where

$A=\frac{a P}{R^{2} T^{2}}$,

$B=\frac{b P}{R T}$,

$Z=\frac{P v}{R T}$,

$a(T)=0.45724 *\left(\frac{R^{2} T_{c}^{2}}{P_{c}}\right) * \alpha\left(T_{r}, \omega\right)$,

$b(T)=0.0778 *\left(\frac{R T_{c}}{P_{c}}\right)$,

$\alpha\left(T_{r}, \omega\right)=\left(1+\left(0.37464+1.54226 \omega-0.26992 \omega^{2}\right) *\left(1-T_{r}^{\frac{1}{2}}\right)\right)^{2}$,

$\mathrm{T}_{\mathrm{c}}, \mathrm{P}_{\mathrm{c}}, \mathrm{T}_{\mathrm{r}}, \mathrm{R}$ and $\omega$ are critical temperature, critical pressure, reduced temperature, universal gas constant and acentric factor, respectively.

The fugacity at experimental pressure and temperature can be obtained from equation (1). Similarly, the fugacity at experimental temperature and hydrate equilibrium pressure can be obtained. Difference between both fugacity values gives fugacity driving force at a particular experimental temperature. 


\section{Tables:}

\section{Table S1}

Summary of all experiments comprising of induction time (IT), gas uptake, time required to reach $90 \%$ completion of the hydrate growth $\left(t_{90}\right)$, and water conversion at $t_{90}$.

\begin{tabular}{|c|c|c|c|c|c|c|c|c|c|c|}
\hline $\begin{array}{l}\text { Exper } \\
\text { iment }\end{array}$ & $\begin{array}{l}\text { Solution } \\
\text { condition* }\end{array}$ & $\mathbf{T}(\mathbf{K})$ & $\begin{array}{l}\mathbf{P} \\
\text { (MPa) }\end{array}$ & $\begin{array}{l}\text { Promot } \\
\text { er } \\
\text { Concen } \\
\text { tration } \\
\text { (ppm) }\end{array}$ & $\begin{array}{l}\text { Fugacity } \\
\text { Driving } \\
\text { force } \\
\text { (MPa) }\end{array}$ & $\begin{array}{l}\text { IT } \\
(\min )\end{array}$ & $\begin{array}{l}\text { Gas } \\
\text { uptake } \\
\text { till IT } \\
\text { [vol/vol } \\
\text { (mol of } \\
\text { gas } \\
\text { /mol of } \\
\text { water)] }\end{array}$ & $\begin{array}{l}\text { Gas } \\
\text { uptake } \\
\text { till IT + } \\
4 \text { hr } \\
\text { [vol/vol } \\
\text { (mol of } \\
\text { gas } \\
\text { /mol of } \\
\text { water)] }\end{array}$ & $\begin{array}{c}\text { t90 } \\
(\mathrm{min})\end{array}$ & $\begin{array}{l}\text { Water } \\
\text { conversion at } \\
\text { t90 }_{90}(\%)\end{array}$ \\
\hline A1 & $\mathrm{F}$ & 273.65 & 3.4 & 0 & 1.4 & 42.3 & $\begin{array}{r}25 \\
(0.021)\end{array}$ & $\begin{array}{r}31.8 \\
(0.027)\end{array}$ & 107.3 & 18.4 \\
\hline A2 & $\mathrm{M}$ & 273.65 & 3.4 & 0 & 1.4 & 7.7 & $\begin{array}{r}22.4 \\
(0.019)\end{array}$ & $\begin{array}{r}24.5 \\
(0.020)\end{array}$ & 115 & 14.2 \\
\hline A 3 & $\mathrm{~F}$ & 273.65 & 3.4 & 0 & 1.4 & 13.7 & $\begin{array}{r}23.9 \\
(0.020)\end{array}$ & $\begin{array}{r}26.8 \\
(0.022)\end{array}$ & 228 & 15.6 \\
\hline B1 & $\mathrm{F}$ & 273.65 & 3.4 & 100 & 1.4 & 9.0 & $\begin{array}{r}21.2 \\
(0.018)\end{array}$ & $\begin{array}{r}112.7 \\
(0.109)\end{array}$ & 172.3 & 70.2 \\
\hline B2 & M & 273.65 & 3.4 & 100 & 1.4 & 85.7 & $\begin{array}{r}26.3 \\
(0.022)\end{array}$ & $\begin{array}{r}29 \\
(0.024)\end{array}$ & 170.0 & 17.0 \\
\hline B3 & $\mathrm{F}$ & 273.65 & 3.4 & 100 & 1.4 & 13.7 & $\begin{array}{r}22.2 \\
(0.018)\end{array}$ & $\begin{array}{r}38 \\
(0.032)\end{array}$ & 52.0 & 21.8 \\
\hline B4 & M & 273.65 & 3.4 & 100 & 1.4 & 42.3 & $\begin{array}{r}26.3 \\
(0.022)\end{array}$ & $\begin{array}{r}31.8 \\
(0.027)\end{array}$ & 169.0 & 18.5 \\
\hline $\mathrm{C} 1$ & F & 273.65 & 3.4 & 300 & 1.4 & 1.3 & $\begin{array}{r}11.6 \\
(0.010)\end{array}$ & $\begin{array}{r}113.8 \\
(0.110)\end{array}$ & 65.3 & 70.5 \\
\hline $\mathrm{C} 2$ & $\mathrm{M}$ & 273.65 & 3.4 & 300 & 1.4 & 1.3 & $\begin{array}{r}12.2 \\
(0.010)\end{array}$ & $\begin{array}{r}109.6 \\
(0.105)\end{array}$ & 82.7 & 67.4 \\
\hline $\mathrm{C} 3$ & $\mathrm{~F}$ & 273.65 & 3.4 & 300 & 1.4 & 16.7 & $\begin{array}{r}26.6 \\
(0.022)\end{array}$ & $\begin{array}{r}115.1 \\
(0.112)\end{array}$ & 55.0 & 72.4 \\
\hline $\mathrm{C} 4$ & $\mathrm{M}$ & 273.65 & 3.4 & 300 & 1.4 & 21.0 & $\begin{array}{r}25.6 \\
(0.021)\end{array}$ & $\begin{array}{r}117.4 \\
(0.115)\end{array}$ & 66.7 & 74.1 \\
\hline D1 & $\mathrm{F}$ & 273.65 & 3.4 & 1000 & 1.4 & 5.7 & $\begin{array}{r}22.9 \\
(0.019)\end{array}$ & $\begin{array}{r}116 \\
(0.113)\end{array}$ & 72.7 & 72.8 \\
\hline
\end{tabular}




\begin{tabular}{|c|c|c|c|c|c|c|c|c|c|c|}
\hline D2 & $\mathrm{F}$ & 273.65 & 3.4 & 1000 & 1.4 & 12.7 & $\begin{array}{r}24.7 \\
(0.021)\end{array}$ & $\begin{array}{r}113.9 \\
(0.110)\end{array}$ & 150.7 & 71.3 \\
\hline D3 & $\mathrm{M}$ & 273.65 & 3.4 & 1000 & 1.4 & 7.3 & $\begin{array}{r}20.6 \\
(0.017)\end{array}$ & $\begin{array}{r}109.6 \\
(0.106)\end{array}$ & 203.0 & 68.0 \\
\hline E1 & $\mathrm{F}$ & 275.65 & 3.6 & 0 & 1.25 & 10.7 & $\begin{array}{r}26.6 \\
(0.022)\end{array}$ & $\begin{array}{r}32.7 \\
(0.028)\end{array}$ & 156.0 & 19.1 \\
\hline E2 & $\mathrm{M}$ & 275.65 & 3.6 & 0 & 1.25 & 7.7 & $\begin{array}{r}24.1 \\
(0.020)\end{array}$ & $\begin{array}{r}30.7 \\
(0.026)\end{array}$ & 173.7 & 17.8 \\
\hline E3 & $\mathrm{F}$ & 275.65 & 3.6 & 0 & 1.25 & 7.7 & $\begin{array}{r}19.4 \\
(0.016)\end{array}$ & $\begin{array}{r}27.3 \\
(0.023)\end{array}$ & 136.0 & 15.6 \\
\hline E4 & M & 275.65 & 3.6 & 0 & 1.25 & 13.3 & $\begin{array}{r}24.8 \\
(0.021)\end{array}$ & $\begin{array}{r}27.1 \\
(0.023)\end{array}$ & 175.0 & 15.8 \\
\hline $\mathrm{F} 1$ & $\mathrm{~F}$ & 275.65 & 3.6 & 100 & 1.25 & 35.7 & $\begin{array}{r}24.1 \\
(0.020)\end{array}$ & $\begin{array}{r}26.8 \\
(0.022)\end{array}$ & 152.0 & 15.6 \\
\hline $\mathrm{F} 2$ & M & 275.65 & 3.6 & 100 & 1.25 & 31.3 & $\begin{array}{r}25.1 \\
(0.021)\end{array}$ & $\begin{array}{r}29.7 \\
(0.025)\end{array}$ & 105.3 & 17.3 \\
\hline F3 & $\mathrm{F}$ & 275.65 & 3.6 & 100 & 1.25 & 39.7 & $\begin{array}{r}27.2 \\
(0.023)\end{array}$ & $\begin{array}{r}111.8 \\
(0.108)\end{array}$ & 153.3 & 69.9 \\
\hline F4 & $\mathrm{M}$ & 275.65 & 3.6 & 100 & 1.25 & 40.0 & $\begin{array}{r}25.1 \\
(0.021)\end{array}$ & $\begin{array}{r}99.7 \\
(0.094)\end{array}$ & 229.3 & 61.1 \\
\hline G1 & $\mathrm{F}$ & 275.65 & 3.6 & 300 & 1.25 & 7.0 & $\begin{array}{r}24 \\
(0.020)\end{array}$ & $\begin{array}{r}116 \\
(0.113)\end{array}$ & 67.0 & 72.9 \\
\hline G2 & $\mathrm{F}$ & 275.65 & 3.6 & 300 & 1.25 & 7.0 & $\begin{array}{r}25.3 \\
(0.021)\end{array}$ & $\begin{array}{r}116.6 \\
(0.114)\end{array}$ & 67.3 & 73.4 \\
\hline G3 & $\mathrm{F}$ & 275.65 & 3.6 & 300 & 1.25 & 6.3 & $\begin{array}{r}18.9 \\
(0.016)\end{array}$ & $\begin{array}{r}108.8 \\
(0.105)\end{array}$ & 63.7 & 67.3 \\
\hline G4 & $\mathrm{M}$ & 275.65 & 3.6 & 300 & 1.25 & 29.3 & $\begin{array}{r}24.9 \\
(0.021)\end{array}$ & $\begin{array}{r}110.4 \\
(0.106)\end{array}$ & 61.3 & 68.8 \\
\hline H1 & $\mathrm{F}$ & 275.65 & 3.6 & 1000 & 1.25 & 6.3 & $\begin{array}{r}23.7 \\
(0.020)\end{array}$ & $\begin{array}{r}111.3 \\
(0.107)\end{array}$ & 68.7 & 69.3 \\
\hline $\mathrm{H} 2$ & $\mathrm{~F}$ & 275.65 & 3.6 & 1000 & 1.25 & 60.7 & $\begin{array}{r}27.6 \\
(0.023)\end{array}$ & $\begin{array}{r}111.3 \\
(0.107)\end{array}$ & 114.0 & 69.6 \\
\hline H3 & $\mathrm{M}$ & 275.65 & 3.6 & 1000 & 1.25 & 91.0 & $\begin{array}{r}25.8 \\
(0.022)\end{array}$ & $\begin{array}{r}109.8 \\
(0.106)\end{array}$ & 56.7 & 68.4 \\
\hline
\end{tabular}




\begin{tabular}{|c|c|c|c|c|c|c|c|c|c|c|}
\hline I1 & $\mathrm{F}$ & 277.65 & 3.8 & 0 & 1.05 & 353.0 & $\begin{array}{r}25 \\
(0.021)\end{array}$ & $\begin{array}{r}30.5 \\
(0.026)\end{array}$ & 50.0 & 17.7 \\
\hline $\mathrm{I} 2$ & M & 277.65 & 3.8 & 0 & 1.05 & 816.3 & $\begin{array}{r}25.2 \\
(0.021)\end{array}$ & $\begin{array}{r}33.8 \\
(0.029)\end{array}$ & 61.3 & 19.6 \\
\hline I3 & $\mathrm{F}$ & 277.65 & 3.8 & 0 & 1.05 & 409.7 & $\begin{array}{r}25.2 \\
(0.021)\end{array}$ & $\begin{array}{r}27.3 \\
(0.023)\end{array}$ & 170.0 & 16.0 \\
\hline I4 & $\mathrm{M}$ & 277.65 & 3.8 & 0 & 1.05 & 459.7 & $\begin{array}{r}24 \\
(0.020)\end{array}$ & $\begin{array}{r}29.8 \\
(0.025)\end{array}$ & 54.0 & 17.2 \\
\hline $\mathrm{J} 1$ & $\mathrm{~F}$ & 277.65 & 3.8 & 100 & 1.05 & 28.7 & $\begin{array}{r}25.2 \\
(0.021)\end{array}$ & $\begin{array}{r}94.7 \\
(0.089)\end{array}$ & 230.3 & 57.7 \\
\hline $\mathrm{J} 2$ & $\mathrm{M}$ & 277.65 & 3.8 & 100 & 1.05 & 157.0 & $\begin{array}{r}25.6 \\
(0.021)\end{array}$ & $\begin{array}{r}31.2 \\
(0.026)\end{array}$ & 100.0 & 18.2 \\
\hline $\mathrm{J} 3$ & $\mathrm{~F}$ & 277.65 & 3.8 & 100 & 1.05 & 193.0 & $\begin{array}{r}26.3 \\
(0.022)\end{array}$ & $\begin{array}{r}28.1 \\
(0.024)\end{array}$ & 164.0 & 16.5 \\
\hline $\mathrm{J} 4$ & M & 277.65 & 3.8 & 100 & 1.05 & 47.0 & $\begin{array}{r}24.3 \\
(0.020)\end{array}$ & $\begin{array}{r}26.3 \\
(0.022)\end{array}$ & 204.0 & 15.3 \\
\hline K1 & $\mathrm{F}$ & 277.65 & 3.8 & 300 & 1.05 & 523.0 & $\begin{array}{r}21.4 \\
(0.018)\end{array}$ & $\begin{array}{r}108.6 \\
(0.104)\end{array}$ & 141.0 & 67.3 \\
\hline $\mathrm{K} 2$ & $\mathrm{M}$ & 277.65 & 3.8 & 300 & 1.05 & 26.3 & $\begin{array}{r}27.2 \\
(0.023)\end{array}$ & $\begin{array}{r}116.4 \\
(0.113)\end{array}$ & 121.3 & 73.3 \\
\hline K3 & $\mathrm{F}$ & 277.65 & 3.8 & 300 & 1.05 & 580.7 & $\begin{array}{r}27.2 \\
(0.023)\end{array}$ & $\begin{array}{r}111.9 \\
(0.108)\end{array}$ & 76.0 & 70.1 \\
\hline $\mathrm{K} 4$ & $\mathrm{M}$ & 277.65 & 3.8 & 300 & 1.05 & 24.0 & $\begin{array}{r}22.9 \\
(0.019)\end{array}$ & $\begin{array}{r}109.4 \\
(0.105)\end{array}$ & 103.0 & 67.9 \\
\hline L1 & $\mathrm{F}$ & 277.65 & 3.8 & 1000 & 1.05 & 1.3 & $\begin{array}{r}14.8 \\
(0.012)\end{array}$ & $\begin{array}{r}111.5 \\
(0.108)\end{array}$ & 67.3 & 69.0 \\
\hline L2 & $\mathrm{M}$ & 277.65 & 3.8 & 1000 & 1.05 & 750.0 & $\begin{array}{r}27.1 \\
(0.023)\end{array}$ & $\begin{array}{r}113.8 \\
(0.110)\end{array}$ & 179.0 & 71.4 \\
\hline L3 & $\mathrm{F}$ & 277.65 & 3.8 & 1000 & 1.05 & 588.7 & $\begin{array}{r}24.9 \\
(0.021)\end{array}$ & $\begin{array}{r}111.4 \\
(0.108)\end{array}$ & 62.3 & 69.5 \\
\hline L4 & M & 277.65 & 3.8 & 1000 & 1.05 & 57.0 & $\begin{array}{r}22.4 \\
(0.019)\end{array}$ & $\begin{array}{r}112.3 \\
(0.109)\end{array}$ & 101.7 & 70.0 \\
\hline
\end{tabular}

* F- Fresh Run and M-Memory Run 


\section{Table S2}

Average $\mathrm{NR}_{15}$ with standard deviation and $\mathrm{R}^{2}$ value for both stages for each experimental condition.

\begin{tabular}{|c|c|c|c|c|c|c|c|c|c|}
\hline \multirow[b]{2}{*}{ Experiment } & \multirow[b]{2}{*}{$\mathrm{T}(\mathrm{K})$} & \multirow[b]{2}{*}{$\begin{array}{l}\text { Pressure } \\
\text { (MPa) }\end{array}$} & \multirow[b]{2}{*}{$\begin{array}{l}\text { L- } \\
\text { tryptophan } \\
(\mathrm{ppm})\end{array}$} & \multicolumn{3}{|c|}{ Stage I NR15 } & \multicolumn{3}{|c|}{ Stage II NR15 } \\
\hline & & & & $\begin{array}{l}\text { Average } \\
(\mathrm{v} / \mathrm{v} / \mathrm{hr})\end{array}$ & $\begin{array}{c}\text { Standard } \\
\text { deviation } \\
(\mathrm{v} / \mathrm{v} / \mathrm{hr})\end{array}$ & $\begin{array}{c}\text { Average } \\
\mathrm{R}^{2}\end{array}$ & $\begin{array}{c}\begin{array}{c}\text { Average } \\
(\mathrm{v} / \mathrm{v} / \mathrm{hr})\end{array} \\
\end{array}$ & $\begin{array}{c}\text { Standard } \\
\text { deviation } \\
(\mathrm{v} / \mathrm{v} / \mathrm{hr})\end{array}$ & $\begin{array}{c}\text { Average } \\
\mathrm{R}^{2}\end{array}$ \\
\hline A1-A3 & 273.65 & 3.4 & 0 & 3.13 & 3.03 & 0.805 & - & - & - \\
\hline B1-B4 & 273.65 & 3.4 & 100 & 10.44 & 4.23 & 0.958 & 35.35 & 0.00 & 0.999 \\
\hline $\mathrm{C} 1-\mathrm{C} 4$ & 273.65 & 3.4 & 300 & 58.80 & 20.42 & 0.848 & 135.49 & 22.97 & 0.995 \\
\hline D1-D3 & 273.65 & 3.4 & 1000 & 14.60 & 0.00 & 0.906 & 96.16 & 28.58 & 0.958 \\
\hline E1-E4 & 275.65 & 3.6 & 0 & 6.98 & 5.01 & 0.893 & - & - & - \\
\hline F1-F4 & 275.65 & 3.6 & 100 & 5.73 & 1.08 & 0.833 & $\begin{array}{l}57.53 \\
\end{array}$ & 15.56 & 0.917 \\
\hline G1-G4 & 275.65 & 3.6 & 300 & 28.32 & 12.98 & 0.856 & 127.99 & 6.30 & 0.995 \\
\hline $\mathrm{H} 1-\mathrm{H} 3$ & 275.65 & 3.6 & 1000 & 126.37 & 15.98 & 0.954 & 38.41 & 28.81 & 0.955 \\
\hline I1-I4 & 277.65 & 3.8 & 0 & 8.97 & 5.64 & 0.898 & - & - & - \\
\hline J1-J4 & 277.65 & 3.8 & 100 & 7.35 & 4.45 & 0.884 & 48.45 & 0.00 & 0.989 \\
\hline K1-K4 & 277.65 & 3.8 & 300 & 21.08 & 16.13 & 0.907 & 64.54 & 25.77 & 0.995 \\
\hline L1-L4 & 277.65 & 3.8 & 1000 & 107.13 & 20.00 & 0.962 & 67.41 & 45.78 & 0.994 \\
\hline
\end{tabular}




\section{Figures:}

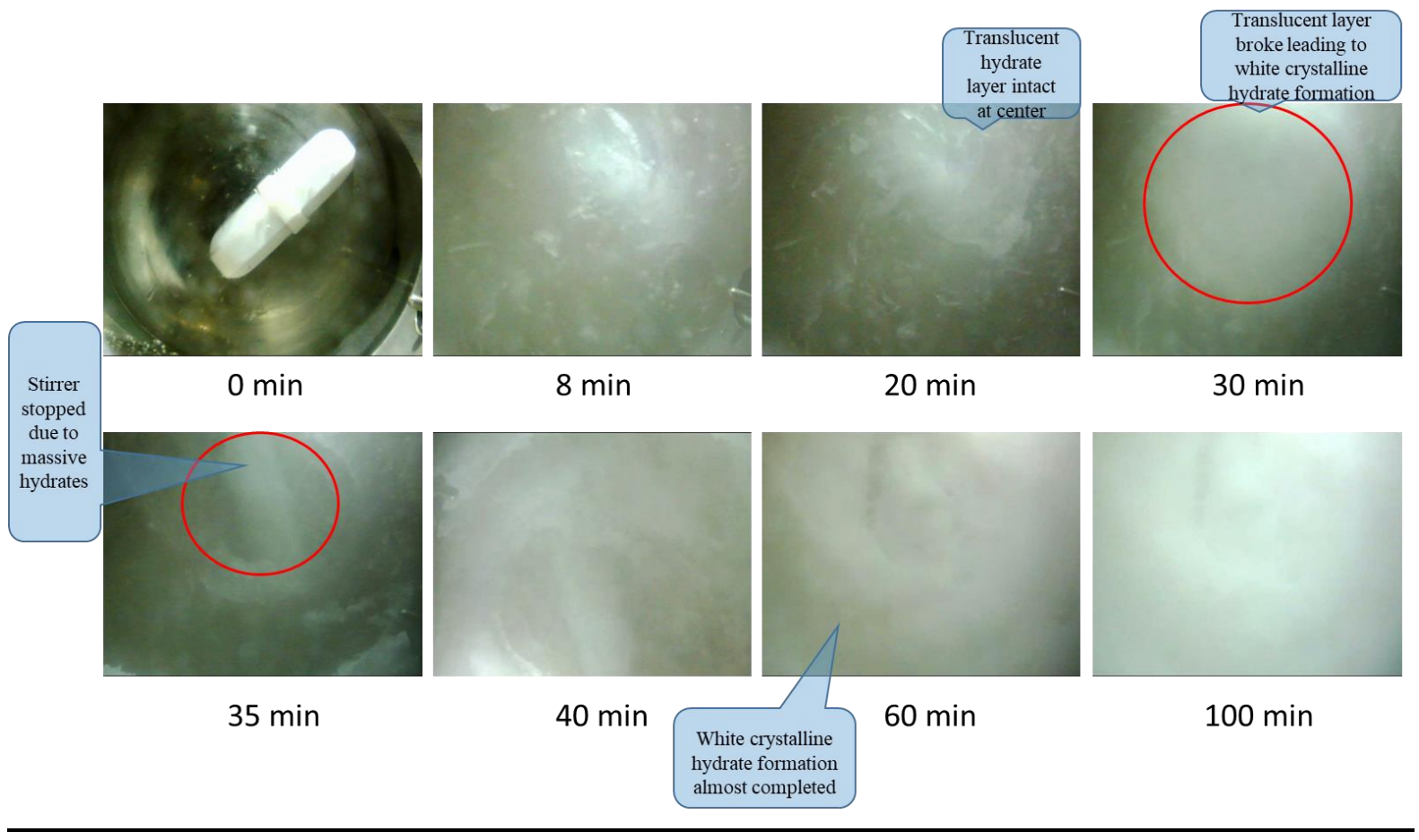

Fig. S1. Top view images for key moments in the experiment showing two stage hydrate formation at 275.65 K, 3.6 MPa and 300 ppm L-tryptophan concentration (Experiment G1). For a better visualization, readers are directed to Video S6 in Supplementary Information. 


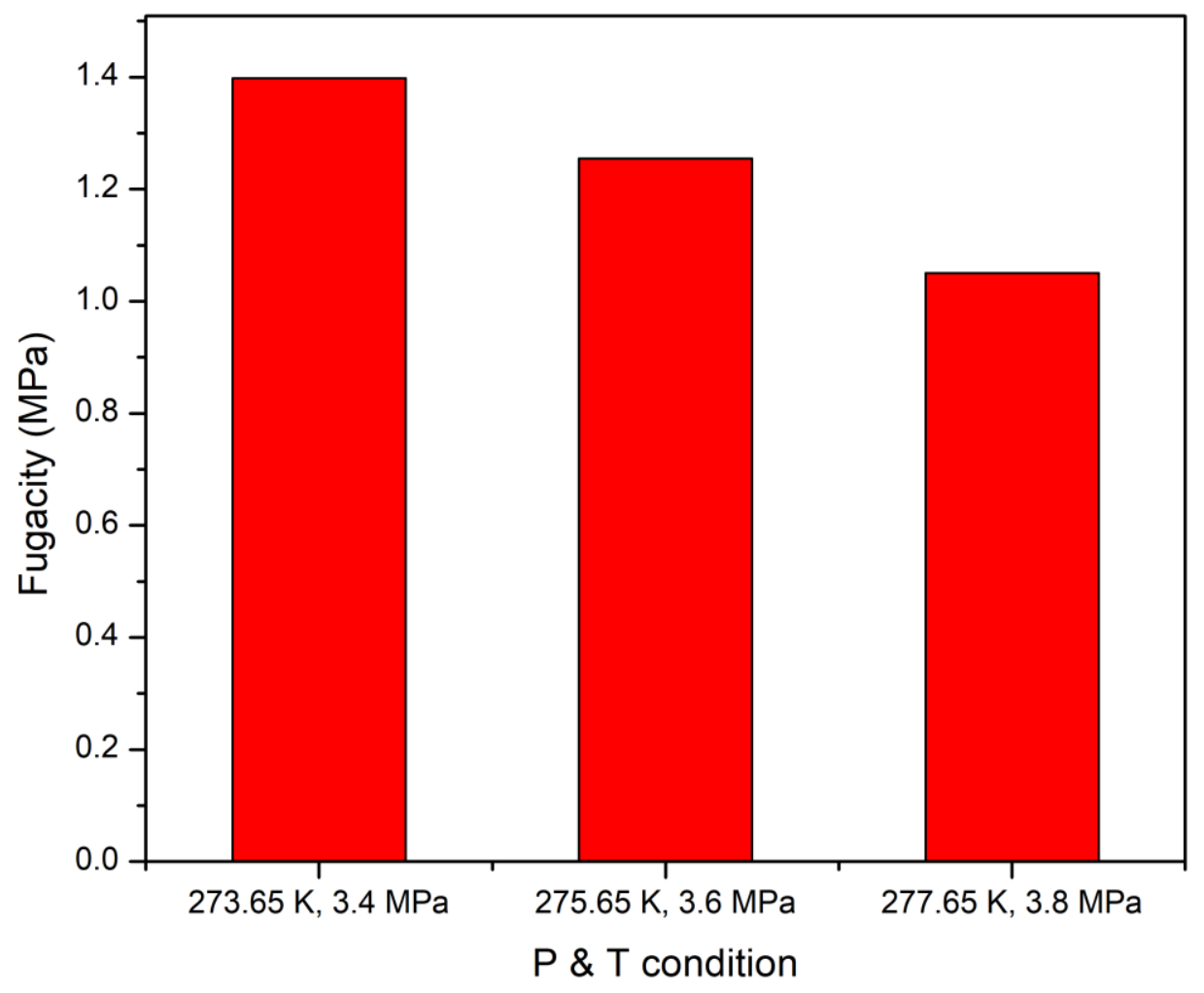

Fig. S2. Fugacity driving force for $\mathrm{CO}_{2}$ hydrate formation at all experimental conditions. 


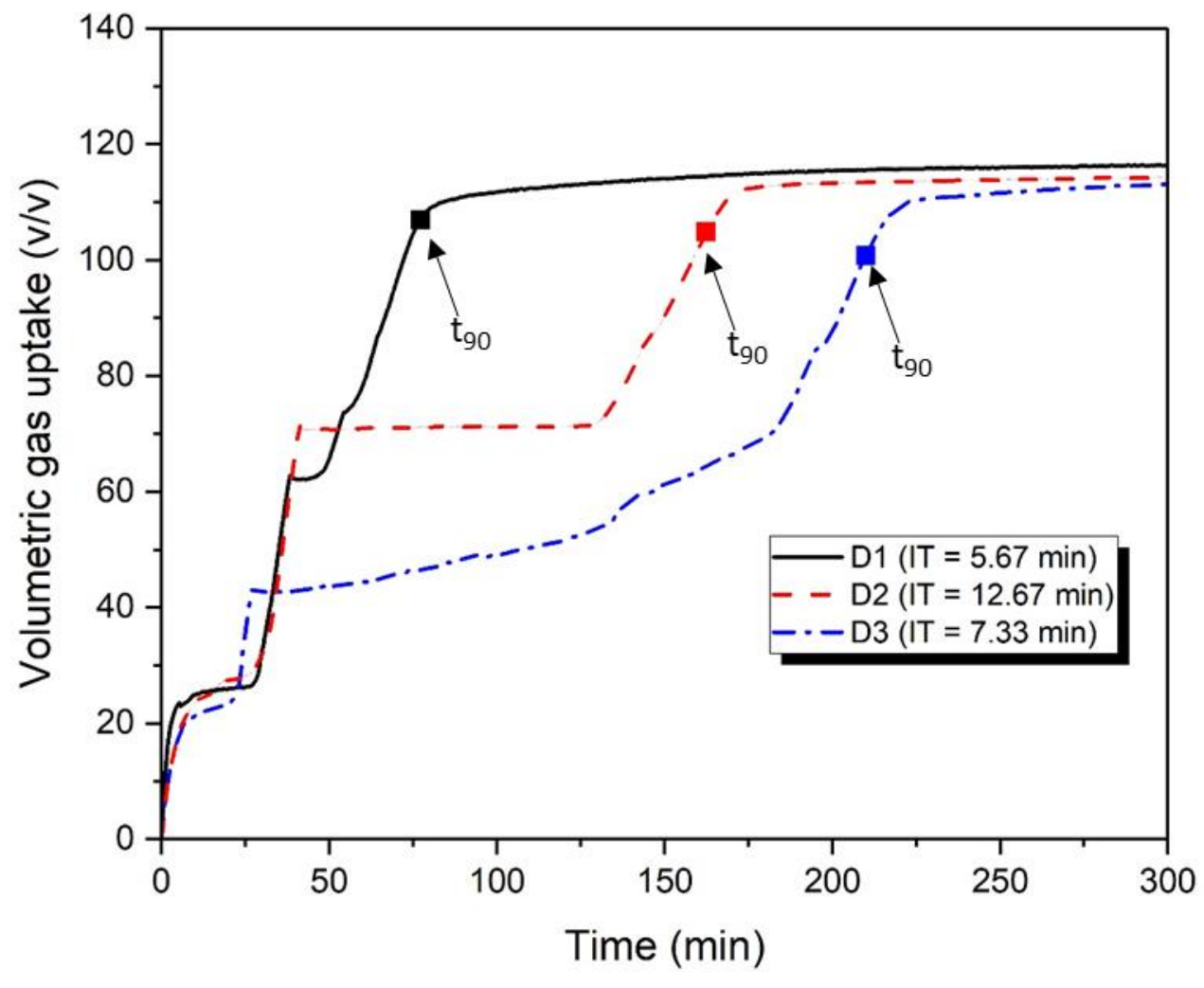

Fig. S3. Gas uptake curves for the experiment conducted at $3.4 \mathrm{MPa}, 273.65 \mathrm{~K}$ and $1000 \mathrm{ppm}$ L-tryptophan concentration. 


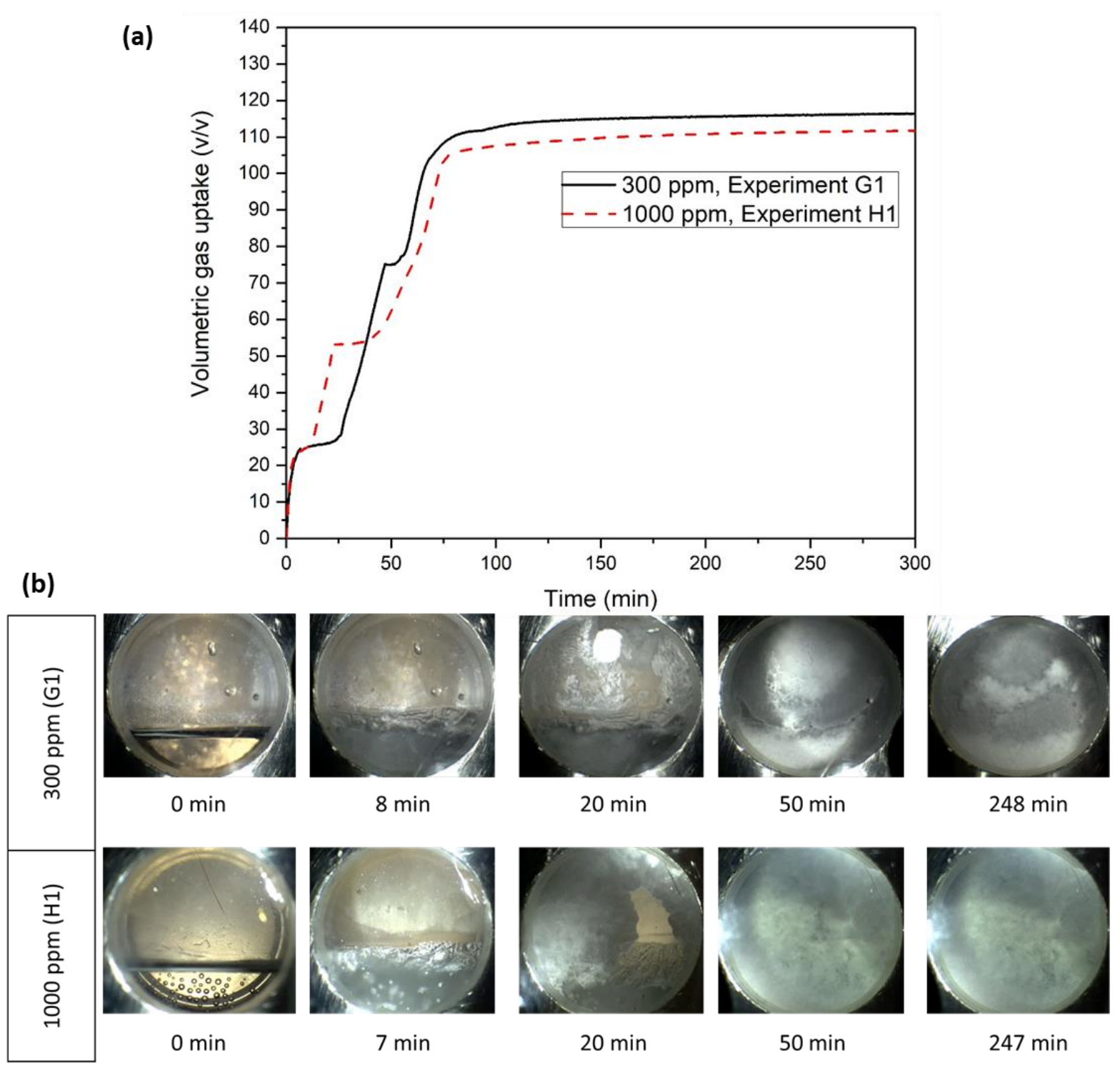

Fig. S4. (a) Comparison of gas uptake between 300 and $1000 \mathrm{ppm}$ and (b) the visual images of the respective experiments at different time periods. 


\section{Supporting Video Captions:}

Video S1: Three stages of $\mathrm{CO}_{2}$ hydrate growth in presence of L-tryptophan as a kinetic promoter [experiment $\mathrm{C} 1$ (300 ppm L-tryptophan, $273.65 \mathrm{~K}$ and 3.4 MPa)] from start to $80 \mathrm{~min}$ (experimental time), the video is sped up by 32 times. Stage (a) time (00:00 - 00:01 of video time), stage (b) time (00:01 - 00:48 of video time) and stage (c) time (00:48 - 02:26 of video time). Massive hydrate formation occurred between 00:59 to 01:50 min in video.

Video S2: Front view video to show nucleation for no-promoter condition [experiment E1 (0 ppm Ltryptophan, $275.65 \mathrm{~K}$ and $3.6 \mathrm{MPa}$ )] from $12 \mathrm{~min}$ to $13 \mathrm{~min}$ (experimental time).

Video S3: Front view video to show formation for no-promoter condition [experiment E1 ( $0 \mathrm{ppm} \mathrm{L-}$ tryptophan, $275.65 \mathrm{~K}$ and 3.6 MPa)] from $13 \mathrm{~min}$ to $71 \mathrm{~min}$ (experimental time), sped up by 30 times. Not much hydrate formation after nucleation can be seen in video.

Video S4: Front view video to show nucleation for promoter condition [experiment G1 (300 ppm Ltryptophan, 275.65 K and 3.6 MPa)] from $7.5 \mathrm{~min}$ to $8.5 \mathrm{~min}$ (experimental time).

Video S5: Front view video to show formation for promoter condition [experiment G1 (300 ppm Ltryptophan, $275.65 \mathrm{~K}$ and $3.6 \mathrm{MPa}$ )] from $8.5 \mathrm{~min}$ to $84 \mathrm{~min}$ (experimental time), sped up by 60 times. Massive hydrate formation occurred between 00:09 to 00:56 (video time).

Video S6: Top view video to show formation for promoter condition [experiment G1 (300 ppm Ltryptophan, $275.65 \mathrm{~K}$ and $3.6 \mathrm{MPa}$ )] from $8.5 \mathrm{~min}$ to $58 \mathrm{~min}$ (experimental time), video sped up by 60 times. Breaking of hydrate layer occurred at around 00:18 min in the video which leads to further massive hydrate growth in the bulk solution till 00:40 min. 